\title{
Atomistic Study of Zwitterionic Peptoid Antifouling
}

\section{Brushes}

David L. Cheung ${ }^{\dagger, *}$ and King Hang Aaron Lau

$\dagger$ School of Chemistry, National University of Ireland Galway, Galway, H91 TK33, Ireland

¥Department of Pure and Applied Chemistry, University of Strathclyde, Glasgow, G1 1XL, UK

* Correspondence emails: david.cheung@nuigalway.ie and aaron.lau@strath.ac.uk

Keywords: Polymer brush, polysarcosine, sarcosine, Nme, biointerface, DOPA, surface grafting, protein adsorption, peptidomimetic, conformation, molecular dynamics, computational chemistry 


\section{ABSTRACT}

Using molecular dynamics (MD) simulations, we study the molecular behavior and hydration properties of a set of antifouling zwitterionic "peptoid" brushes, grafted on a rutile surface, that has been previously reported to exhibit excellent resistance against protein adsorption and cell attachment. Peptoids are novel poly $(N$-substituted glycine $)$ peptide mimics with sidechains attached to amide nitrogens. They constitute a unique model polymer system because hundreds of sidechains have been demonstrated, and the exact chain length and sequence order of the residues/monomers may be specified in experiments. In this report, we vary the brush grafting density as well as the sidechain/polymer molecular volume. We include in our study polysarcosine as an uncharged comparison with a small polymer chain cross-section. Sarcosine is the simplest peptoid residue with only a nominally hydrophobic methyl group as sidechain but is also reported to exhibit high antifouling performance. Overall, we show in detail how molecular volume and hydration effects are intertwined in a zwitterionic polymer brush. For example, the zwitterionic design significantly promotes extended chain conformations and could actually lower the overall electrostatic potential. Some properties promoted by the balanced charges, such as chain flexibility and hydration, increase more prominently at "low" to "intermediate" chain densities. These and other observations should provide insight on the molecular behavior of peptoids and inform the design of zwitterionic antifouling polymer brushes. 


\section{Introduction}

Biofouling of material surfaces by the non-specific attachment of biomolecules, biological cells, and organisms can have very costly consequences, from infection of implanted biomedical devices and opsonization of nanotherapeutics, to the fouling of marine and engineering surfaces. ${ }^{1,2}$ Antifouling surface-grafted polymer brushes are aimed primarily at preventing protein adsorption, which mediate many of the process that lead to further fouling by cells and organisms. ${ }^{1,3}$ They are commonly formed by grafting one of the ends of a linear swollen polymer to the surface, which causes, on average, extension of the polymer chains away from the surface-hence the brush description - if the polymer molecular weight and areal chain density are sufficiently high. Foundational studies have shown how adsorption of biomolecules/proteins would require expulsion of the water hydrating the polymer and restriction of thermal fluctuations of the chains, both of which are unfavorable for highly flexible and water-soluble polymers. ${ }^{4-6,7}$ Other pioneering work have highlighted the importance of selecting appropriate chemical designs of a surface or polymer for reducing the intermolecular interactions with proteins, in order to prevent protein adsorption..$^{6,8-10}$

Zwitterionic polymer brushes have been shown to have excellent antifouling properties. ${ }^{1,7,11-16}$ These brushes may be constituted from different betaine derivatives ${ }^{9,13,14,17}$ as well as from mixtures of anionic and cationic groups, which offer some synthetic convenience and flexibility. ${ }^{15,18-20}$ The mixed brushes may also be advantageous in providing reactive groups for further surface functionalization and stimuli-responsive behavior. ${ }^{2,2}{ }^{22}$ Relatively thick brushes may be grafted using controlled radical polymerization from surface-immobilized initiators, although control of polydispersity and grafting density for high antifouling performance requires synthetic care. ${ }^{16,23}$ Tightly controlled polymers pre-formed with a surface anchor group may also 
be grafted to a surface, but these brushes may be limited in molecular weight to enable efficient surface anchoring and hence require relatively high grafting densities to be effective. ${ }^{18}$

Although recent work has continued to expand the potential applications of zwitterionic surface brushes from biomedical to membrane filtration and marine antifouling, ${ }^{24-28}$ a clear advantage compared to other polymer designs is not always observed. ${ }^{24,29-31}$ The excellent antifouling properties of zwitterionic polymer brushes are frequently attributed to their high hydration by charge groups. 2, 7, 13, 32-34 The water layer around highly hydrated phosphobetaine polymers has also been observed to display different dynamics from that around comparably hydrophilic polymers ${ }^{35}$ which is hypothesized to be related to improved biomedical performance. ${ }^{7,35}$ Details regarding the chemical structure of the zwitterionic groups, such as the separation between the oppositely charged groups, are also known to significantly alter the number and spatial arrangement (i.e. structuring) of water molecules hydrating the polymer. ${ }^{12,36-}$ ${ }^{38}$ Overall, an improved understanding of the molecular behavior of zwitterionic polymer brushes should enable better design of such antifouling surfaces.

To address this, we employ atomistic molecular dynamics with explicit water to computationally study the hydration and molecular behavior of mixed charge zwitterionic "peptoid" polymer brushes. Peptoids are novel peptide analogs with sidechains attached to the backbone amide nitrogens instead of the $\alpha$-carbons. ${ }^{39,40}$ This sidechain shift leaves behind a polar and water soluble poly( $\mathrm{N}$-substituted glycine) backbone with only H-bond acceptors, resulting in an achiral and highly flexible polymer with behavior largely dependent on sidechain properties and that does not form intra-backbone H-bonds. ${ }^{41,42}$ Peptoids may be synthesized by an efficient solid phase protocol to obtain exact chain lengths and specific monomer sequences. ${ }^{39,43}$ Mimics of all 20 natural amino acids as well as a few hundred other residues with non-natural sidechains 
have been demonstrated. These attributes make peptoids an unparalleled experimental model system for investigating polymer sidechain composition and sequence design..$^{39,44,45}$ In addition, atomistic simulation parameters for peptoids have recently become available. ${ }^{46,47}$ We therefore base our study on peptoid brushes that have previously been shown experimentally to exhibit excellent antifouling properties. ${ }^{18,}{ }^{48-52}$ In particular, we simulate the behavior of a zwitterionic peptoid containing pairs of residues with oppositely charged sidechains interspaced between uncharged residues, which was previously shown to exhibit lower protein adsorption and E. coli attachment than an uncharged counterpart at low to intermediate grafting densities. ${ }^{18}$

Special attention is paid to the balance between charge, hydration, and molecular volume and chain density effects. We compare the zwitterionic sequence with its uncharged counterpart, and also compare the use of two different uncharged residues $-\mathrm{N}$-methylglycine (a.k.a. sarcosine, the simplest peptoid) and the larger N-methoxyethylglycine (with sidechains that mimic the polyethylene glycol repeating unit). By also varying the grafted chain density and quantifying the water and brush structures with a range of representative parameters, we aim to uncover how incorporation of a zwitterionic charge design, together with molecular volume effects, could together control chain flexibility and brush extension as well as charge complexation and hydration. Although simulations of protein adsorption were not performed, the brush properties observed are expected to directly impact on antifouling behavior, and our results should inform the design and behavior of zwitterionic antifouling polymer brush surfaces in future studies.

\section{Simulation details}

\subsection{Peptoid sequence design}

Our MD study is based on the uncharged 20-mer PS (polysarcosine) and PM brushes previously reported to exhibit excellent antifouling properties (Figure 1 ). ${ }^{48-50,53}$ PS and PM are 
composed of sarcosine (Sar) and N-methoxyethyl glycine (Nme) residues, respectively. Sar is the simplest peptoid monomer/residue with a single methyl group as sidechain while the Nme methoxyethyl sidechain is significantly larger, and mimics the terminus of antifouling methoxyterminated polyethylene glycol (PEG). The poly( $N$-substituted glycine) backbone of peptoids, with multiple H-bond accepting oxygen and nitrogen atoms, is inherently hydrophilic.

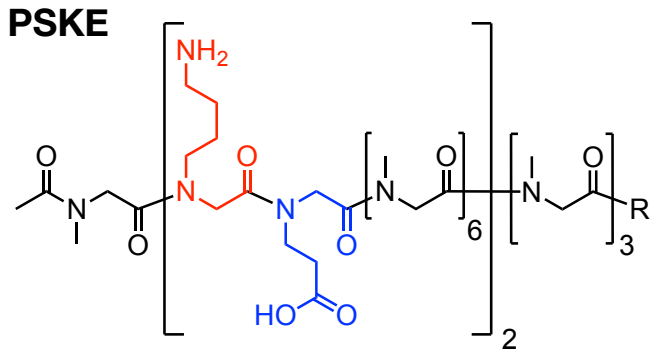

Ac-Sar-(Nlys-Nglu-Sar 6 )-Sar ${ }_{3}-\mathrm{R}$

PS<smiles>[R]C(=O)CN(C)C(C)=O</smiles>

Ac-Sar $_{20}-\mathrm{R}$
PM

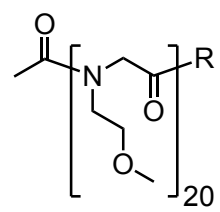

$\mathrm{Ac}^{-\mathrm{Nme}_{20}-\mathrm{R}}$

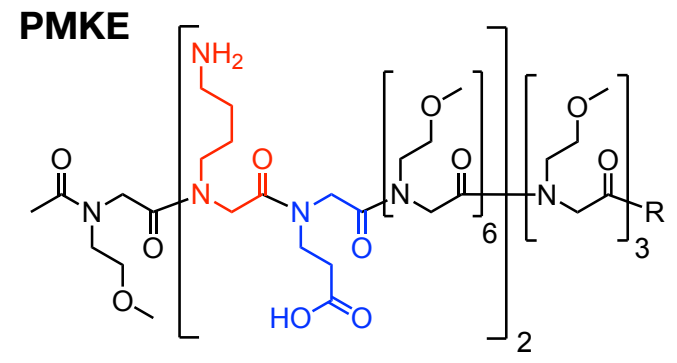

Ac-Nme-(Nlys-Nglu-Nme $\left.{ }_{6}\right)-\mathrm{Nme}_{3}-\mathrm{R}$

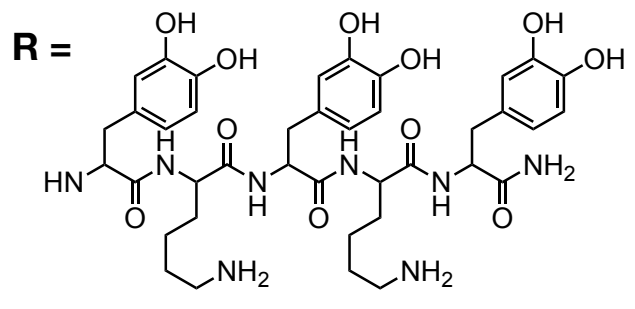

DOPA-Lys-DOPA-Lys-DOPA-Am

Figure 1 Chemical structures of zwitterionic and uncharged peptoids. The corresponding monomer/residue sequences are specified under the structures of each species. See main text for explanation of the naming convention. The $\mathrm{R}$ group is a pentapeptide conferring surface adhesion as reported in the literature.

The zwitterionic PMKE and PSKE peptoid sequences possess pairs of oppositely charged residues, Nlys and Nglu, that are direct analogues of the amino acids lysine $(\mathrm{K})$ and glutamic acid (E), inserted into the PM and PS chains. The pairs of Nlys and Nglu are positioned near the 
$\mathrm{N}$-termini and in the middle of the chains (residues 2/3 and 10/11 counting from the N-terminus). The acetyl-capped N-terminus and the "outermost" residue 1 are always uncharged. In experiments, PMKE showed improved resistance against protein adsorption and E. coli attachment compared to PM brushes at low grafting densities. ${ }^{18}$ As Nme, Nlys and Nglu residues have very similar molecular volumes, comparison of PM and PMKE should reveal changes in brush structure and hydration directly due to the zwitterionic charged design. Although PSKE has not previously been reported, its behaviour as revealed by MD should point to the effects of modifying the polymer (side)chain molecular volume on the properties of zwitterionic brushes.

\subsection{MD methodology}

In mimicking the relevant experimental systems, the peptoid brush surfaces were constructed by placing a regular array of fully extended peptoid molecules on a rutile surface (with the Cterminal DOPA anchoring group close to the surface). The underlying rutile $\left(\mathrm{TiO}_{2}(110)\right)$ surface has dimensions $72.14 \AA$ and $74.4 \AA$ (11 and 25 unit cells) in the $x$ and $y$ directions. In the $z$ direction the slab thickness was $16 \AA$. The length of the simulation box in the $\mathrm{z}$ direction was $140 \AA$. Following the strategy reported in the literature, ${ }^{54}$ peptoid chains are constrained to the surface by fixing the $z$ coordinate of $\mathrm{H} \zeta$ atom in the terminal DOPA residue to $1.78 \AA$ above the highest atom in the rutile surface (corresponding to the minimum in the Lennard-Jones potential between these atoms). These atoms were free to move in the xy-plane, allowing the peptoid chains to adopt their preferred arrangement across the surface and simulating the initial surface binding of the DOPA to $\mathrm{TiO}_{2}$. The surfaces were solvated with water molecules (numbers of water molecules for each system listed in Table 1). Chloride ions were added to neutralise lysine (lys) residues in the DOPA-lys pentapeptide surface anchoring segment, with 12, 40, and 60 ions added to the systems with number of peptoid chains in the array $\left(\mathrm{N}_{\text {chain }}\right)$ set at 6, 20 and 30, 
respectively. The system is periodic in the $x$ - and $y$-directions, while it is confined between repulsive $\mathrm{LJ}$ walls in the $z$-direction.

$\mathrm{N}_{\text {chain }}=6,20$ and 30 correspond to grafting densities of $0.11 \mathrm{~nm}^{-2}, 0.37 \mathrm{~nm}^{-2}$ and $0.56 \mathrm{~nm}^{-2}$, respectively. In comparison, the experimentally determined chain densities for preventing $>95 \%$ of serum protein adsorption is $\sim 0.3 \mathrm{~nm}^{-2}$ for the PM and PMKE brushes, ${ }^{18}$ and the "critical" density for essentially complete prevention of fibrinogen adsorption is $\sim 0.5 \mathrm{~nm}^{-2}$ for PS, PM and PMKE brushes. ${ }^{18,49,50}$ Thus the lowest density studied is expected to be below the density needed for antifouling for all brush species, and the intermediate and highest densities are close to and above the critical density, respectively.

Table 1 Number of water molecules for each simulated system

\begin{tabular}{|l|l|l|l|}
\hline & $\begin{array}{l}\sigma=0.11 \mathrm{~nm}^{-2} \\
\left(\mathrm{~N}_{\text {chain }}=6\right)\end{array}$ & $\begin{array}{l}\sigma=0.37 \mathrm{~nm}^{-2} \\
\left(\mathrm{~N}_{\text {chain }}=20\right)\end{array}$ & $\begin{array}{l}\sigma=0.56 \mathrm{~nm}^{-2} \\
\left(\mathrm{~N}_{\text {chain }}=30\right)\end{array}$ \\
\hline PM & 20721 & 18536 & 16993 \\
\hline PMKE & 20693 & 18408 & 16819 \\
\hline PS & 20979 & 19380 & 18297 \\
\hline PSKE & 20901 & 19140 & 17944 \\
\hline
\end{tabular}

The interactions were modelled using the Charmm27 force field.$^{55}$ Parameters for the Nme and Sar residues were taken from Mirijanian et al. ${ }^{47}$ Parameters for the Nlys and Nglu sidechains were taken from the corresponding amino acids (Lys and Glu), with the backbone parameters taken from Sar residues. The DOPA residues were modelled using parameters taken from the Swiss Sidechain database. ${ }^{56}$ Water was modelled using the modified TIP3P ${ }^{57}$ model and $\mathrm{TiO}_{2}$ was modelled using the potential of Předota $e t a l .^{58}$ 
Van der Waals interactions were evaluated with a cut off of $12 \AA$, with corrections to the energy and pressure applied. Electrostatic interactions were evaluated using a particle-mesh Ewald sum, ${ }^{59}$ with a real space cut off of $12 \AA$ and a reciprocal space grid of $48 \times 48 \times 280$.

All simulations were performed using the Gromacs ${ }^{60,61}$ molecular dynamics package (version 5.0), with standard Gromacs utilities used to construct the systems. The system was energy minimised using the steepest descents algorithm, followed by short (10 ps) NVT simulations. Simulations were performed at $298 \mathrm{~K}$, with temperature controlled using the velocity rescaling $\operatorname{algorithm}^{62}$ with a relaxation time of 0.1 ps. Simulations of 100 ns were performed (with a timestep of $2 \mathrm{fs}$ ), with coordinates saved every $10 \mathrm{ps,}$, giving a total simulation time of $1.2 \mu \mathrm{s}$ across all systems. Analysis of the simulations was performed using standard Gromacs utilities, in-house scripts using the MDAnalysis ${ }^{63}$ package, and VMD. ${ }^{64}$ Only the last 50 ns of each simulation was used for the analysis.

\section{Results}

\subsection{Brush structure}

Qualitative information on the structure of the brushes can be obtained from simulation snapshots (Figure 2). An increase in density and thickness of the layer with the increase in peptoids can be clearly seen in all cases, along with the location of the charged residues on the outside of the brush. From top-down views it can be seen that at the lowest density $\left(0.11 \mathrm{~nm}^{-2}\right)$ the surface is not uniformly covered by peptoids. Rather these chains cluster together leaving significant voids on the surface that are persistent over the $100 \mathrm{~ns}$ timescale of the simulations (see Supplementary videos 1-4), potentially allowing adsorption of proteins which are (or have domains that are) small enough to access. Nonetheless this effect is mitigated by the dynamic 
thermal fluctuation of the chains. Thus experimentally, we have observed some protein adsorption at this chain density, but at a much lower level than without the peptoids. ${ }^{18,49,50}$ For the intermediate density $\left(0.37 \mathrm{~nm}^{-2}\right)$ the PS surface still has a significant amount of exposed surface, while for the other systems the surface is largely covered (Supplementary videos 5-8).

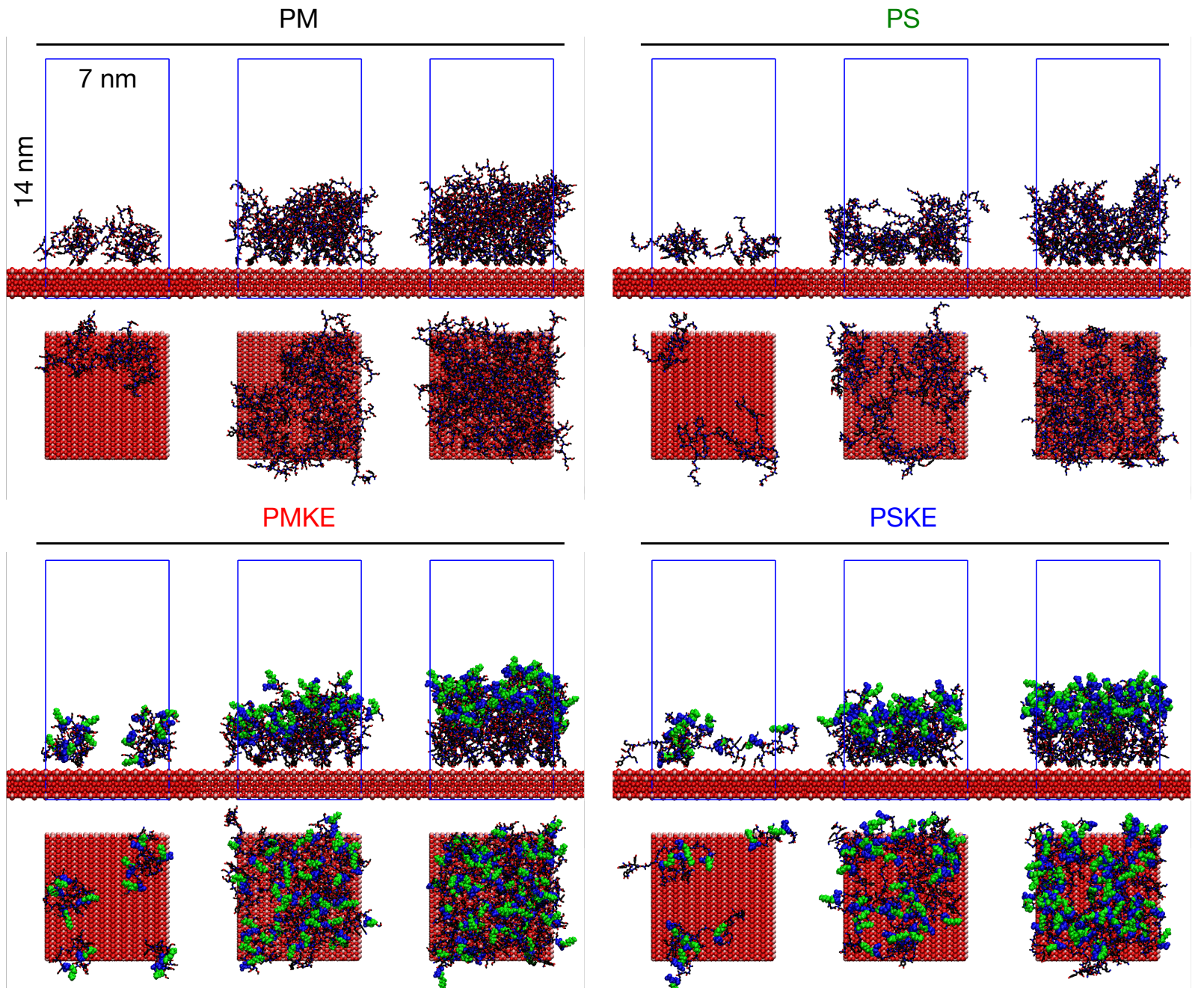

Figure 2 Simulation snapshots of PM (top left), PS (top right), PMKE (bottom left), and PSKE (bottom right) peptoid brushes, showing grafting densities $\sigma=0.11 \mathrm{~nm}^{-2}, 0.37 \mathrm{~nm}^{-2}$, and $0.56 \mathrm{~nm}^{-2}$ (left to right within each category of chains). For PMKE and PSKE, Nglu and Nlys residues are shown in blue and green, respectively. 
At the highest density $\left(0.56 \mathrm{~nm}^{-2}\right)$ the surface is more uniformly covered and the brush top surface is relatively uniform for the PM and PMKE brushes. However long-lived undulations, or layer imperfections, are found for the PS/PSKE brushes, especially for the uncharged PS (Supplementary videos 9-12). A measure of these undulations is the standard deviation of the zposition of the heavy atom in each peptoid furthest from the surface - the values for PS/PSKE are larger than the corresponding brushes with Nme sidechains (e.g. $8.6 \pm 0.5 \AA$ (PS) vs. $6.8 \pm$ $0.7 \AA(\mathrm{PM})$ at $0.37 \mathrm{~nm}^{-2}$ and $6.7 \pm 0.6 \AA$ (PS) vs. $6.5 \pm 0.6 \AA(\mathrm{PM})$ at $0.56 \mathrm{~nm}^{-2}$; see ESI Table $\mathrm{S} 1$ for all layers). The long-lived undulations could indicate that, due to the lower molecular volume of the Sar sidechain, the higher chain density at $0.56 \mathrm{~nm}^{-2}$ is still not sufficient to enforce chain extension away from the surface, and certain conformations of the peptoid chain may persist without some other forcing. Indeed, we speculate that this "open" structure could contribute to the relatively low contact angles observed for the uncharged PS brush $\left(\sim 15^{\circ}\right.$ advancing and $\sim 6^{\circ}$ receding at $\left.0.56 \mathrm{~nm}^{-2}\right),{ }^{50}$ given the peptoid backbone's hydrophilic nature and the large number of water molecules allowed within the PS structure (see section 3.2).

More quantitatively, the chain density profiles for the peptoid brushes are shown in Figure 3(atop). Clear differences between the brushes containing Nme and Sar residues (i.e. between PM/PMKE and PS/PSKE, respectively) can be seen for the lower density, with the Nme containing brushes being both denser and more extended. This arises due to the greater molecular volume of the Nme methoxyethyl sidechain compared to the Sar methyl sidechain. Conversely, the profiles for PM and PMKE are very similar to each other, reflecting the fact that the Nlys and Nglu residues are similar in molecular volume as the Nme sidechains. ${ }^{18}$ Nonetheless, at the highest chain density $\left(0.56 \mathrm{~nm}^{-2}\right)$, a small expansion of the PMKE profile compared to that for PM can be seen around $80 \AA$, indicating the independent effects of the 
zwitterionic charges. In comparison, a larger difference is seen at all densities for the Sar containing brushes, where PSKE is more extended than the neutral PS due to the larger size difference between the Sar and charged sidechains, in addition to the effects of the charged residues.

Density profiles highlighting the charged residues (Figure 3(a)-bottom) indicate the cause of the chain expansion for the zwitterionic peptoids (PMKE and PSKE). Consistent with the snapshots (Figure 2), the charged Nlys and Nglu residues are clearly skewed towards the outside of the brush, in contact with the water. While these distributions are relatively broad, the Nlys residues of PMKE are found further from the surface than the Nglu residues at all densities, even though they participate less in H-bonding than Nglu sidechains (see section 3.2). This allows the larger Nlys sidechains to increase their free volume by partitioning into the water. For PSKE this effect is only seen at 0.11 and $0.37 \mathrm{~nm}^{-2}$, probably reflecting the fact that free volume effects is more important for PMKE brushes that incorporate the larger Nme residues.

The extension of the chains may be quantified through the average chain end-to-end distance, $r_{e e}$ (Figure 3 (b)). Typically $r_{e e}$ increases with chain density. The largest change in $r_{e e}$ from 0.11 to $0.56 \mathrm{~nm}^{-2}$ is found for the zwitterionic PMKE, while the uncharged PM counterpart exhibits slightly smaller changes. These increase in $r_{e e}$ are also reflected in the density profiles (Figure 3(a)) and simulation snapshots (Figure 2). In comparison, the uncharged PS with much smaller Sar residues show relatively little increase in $r_{e e}$ with chain density. Notably, the increases for its zwitterionic counterpart PSKE tracks more closely with the Nme-based PM and PMKE than with PS, indicating that the influence of the zwitterionic charge design can be as large as molecular volume effects for $r_{e e}$. 


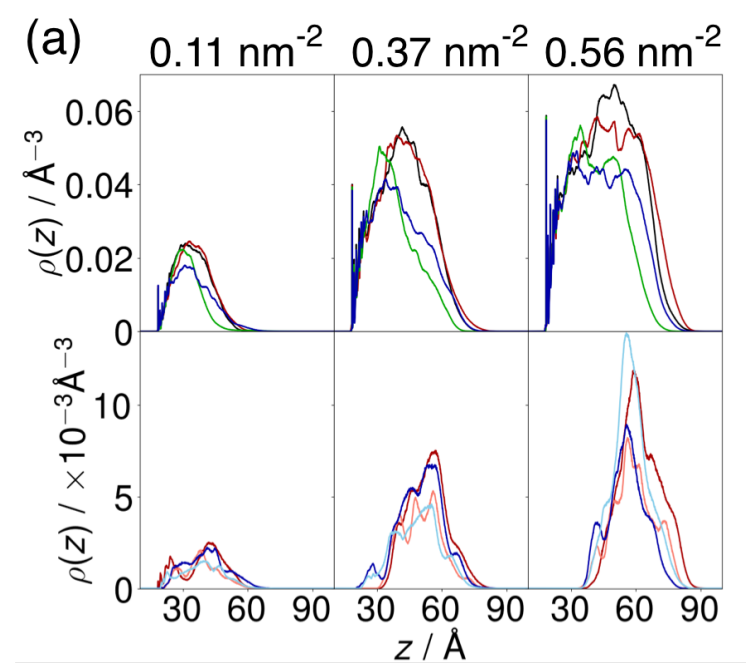

(b)

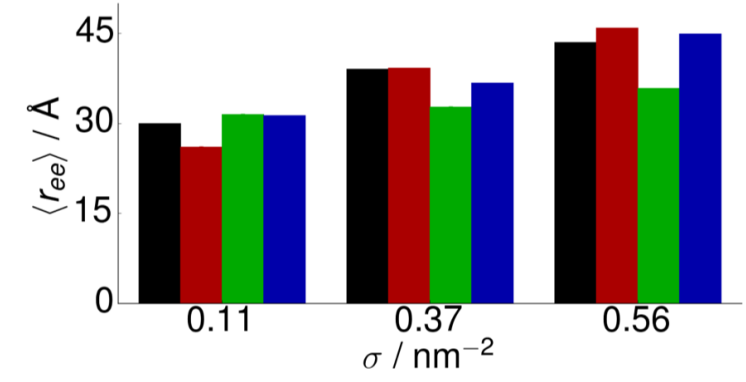

(c)

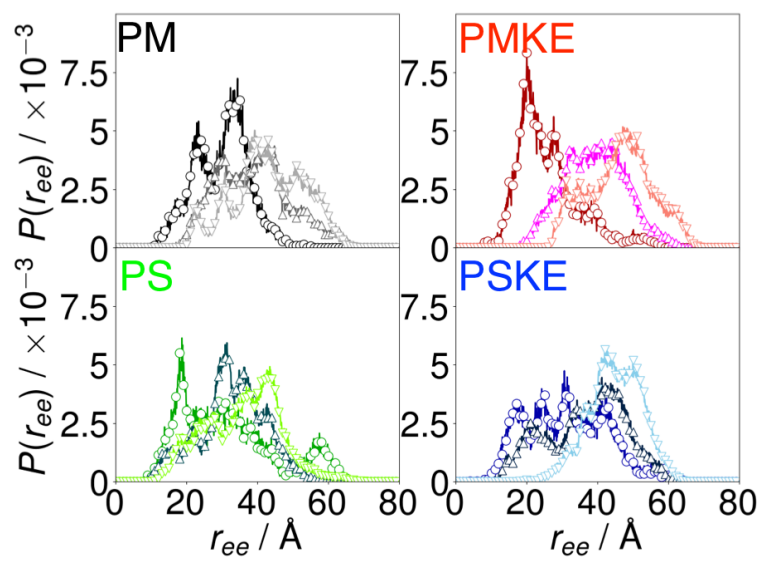

Figure 3. Structure of peptoid brushes. (a) Density profile for the brush (top) and for only the charged peptoid residues (bottom) for (left to right) $\sigma=0.11 \mathrm{~nm}^{-2}, 0.37 \mathrm{~nm}^{-2}$, and $0.56 \mathrm{~nm}^{-2}$ brushes. In the top row black, red, green, and blue denote PM, PMKE, PS, and PSKE brushes respectively. In the second row red, pink, blue, and pale blue denote PMKE (Nlys), PMKE 
(Nglu), PSKE (Nlys), and PSKE (Nglu). (b) Average end-to-end length for PM (black), PMKE (red), PS (green), and PSKE (blue) brushes. (c) End-to-end length probability histograms for PM (top left), PMKE (top right), PS (bottom left), and PSKE (bottom right) brushes. $\sigma=0.11 \mathrm{~nm}^{-2}$, $0.37 \mathrm{~nm}^{-2}$, and $0.56 \mathrm{~nm}^{-2}$ brushes denoted by black, dark grey, grey (PM), red, magenta, pink (PMKE), green, dark green, light green (PS), and blue, dark blue, and light blue (PSKE).

The end-to-end probability distributions, $P\left(r_{\text {ee }}\right)$ (Figure 3(c)) provide more information on the chain extension of the chains. For the Nme-based brushes more extended conformations become more common as the grafting density increases and the peptoid conformations become more constrained. Notably, even though the largest $r_{\text {ee }}$ length only increases slightly as the chain density increases, the probability of more extended chains significantly increases, especially for the charged brushes (e.g. clear shifts of the centres of distribution from 40 to $50 \AA$ for PMKE and from 35 to $45 \AA$ for PSKE, as $\sigma$ increases from 0.37 to $0.56 \mathrm{~nm}^{-2}$ ).

Interestingly, Sar containing brushes (PS and PSKE) were extended even at the lowest grafting density (i.e. relatively flat distributions in $r_{\text {ee }}$ spanning 10 to $60 \AA$, including a peak for PS at $r_{e e} \sim$ $60 \AA$ ). This corresponds to chains that are extended in the plane of the surface suggesting that the Sar residues may interact more strongly with the underlying rutile surface than the Nme residues, or that the smaller molecular volume of the Sar residues leaves sufficient openings/voids for such surface interactions at low chain grafting densities. This extension of PS chains might explain the similar abilities of PS and PM to resist protein adsorption at low chain densities (similar critical antifouling densities previously observed) ${ }^{18,49,50}$ even though the snapshots (Figure 2) show significant unevenness of the PS grafted structures. 


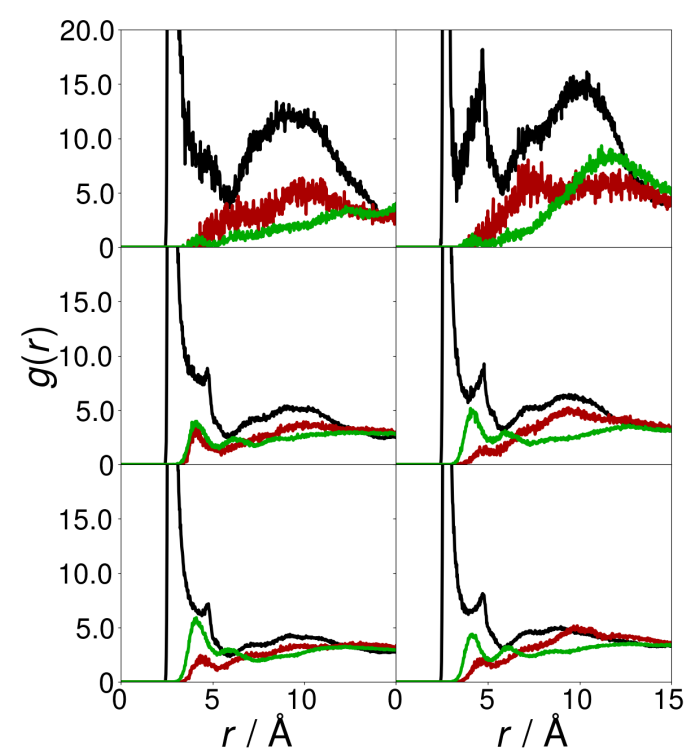

Figure 4 Radial distribution functions involving $\mathrm{N} \zeta$ (Nlys) and $\mathrm{O} \varepsilon$ (Nglu) atoms. Left and righthand panels show RDFs for PMKE and PSKE for (top to bottom) $\sigma=0.11 \mathrm{~nm}^{-2}, 0.37 \mathrm{~nm}^{-2}$, and $0.56 \mathrm{~nm}^{-2}$. Black, red, and green denote $\mathrm{g}_{\mathrm{NZ}-\mathrm{OE}}, \mathrm{g}_{\mathrm{NZ}-\mathrm{NZ}}$, and $\mathrm{g}_{\mathrm{OE}-\mathrm{OE}}$ respectively.

The intrinsic flexibility of the peptoid backbone regardless of the sidechain design ${ }^{42}$ appears to allow for a significant degree of complexation between the charged residues, as examined through the radial distribution functions between the sidechain nitrogen (in Nlys) and oxygen atoms (in Nglu; Figure 4). These show a large peak at around $3 \AA$, corresponding to the close contact between oppositely charged atoms. There are also a number of additional peaks, showing longer-ranged ordering. This is strongest for the lowest grafting density, where there is a broad secondary peak in the RDF around $10 \AA$ for both PMKE and PSKE. PSKE also has a sharp peak around $4 \AA$. As the grafting density increases the ordering beyond the first peak becomes weaker, with only a single secondary peak (at approximately $5 \AA$ A) being present. This may correspond to 
the second $\mathrm{O} \varepsilon$ atom in the Nglu sidechain, and is stronger for the PSKE brush, which may be due to the smaller Sar residues making these more flexible compared to the bulkier Nme sidechains.

At low grafting densities there is little correlation between like charge atoms $(\mathrm{N} \zeta-\mathrm{N} \zeta$ and $\mathrm{O} \varepsilon$ $\mathrm{O \varepsilon}$ ). As the grafting density increases a peak appears in the RDFs for the like charged atoms around $r \sim 4 \AA$ (note that Oع atoms on the same residue are excluded from the calculation of $\mathrm{g}_{\mathrm{OE}}$ $\mathrm{OE})$. This is at a larger separation than in the $\mathrm{g}_{\mathrm{NZ}-\mathrm{OE}}$ so it corresponds to $\mathrm{O} \varepsilon$ atoms on different residues interacting with the same $\mathrm{N} \zeta$ atom (and vice versa). This pairing of ions acts to reduce the electrostatic potential compared to the non-zwitterionic brushes.

The electrostatic potential calculated for the peptoid brushes are shown in Figure 5. At lower grafting density the potentials for the different brushes are qualitatively similar. Notably, due to the ion pairing, the potential for the zwitterionic brushes are lower than for the neutral brushes. As the grafting density increases the potential becomes more complex and no longer decays monotonically with increasing height, in particular with a shoulder in the potential appearing inside the zwitterionic brushes at around a height of $60 \sim 80 \AA$. This arises due to the charged residues partitioning to the brush surface located at these heights. For the PMKE brush the surface localization of the potential is most pronouced as chain density increased to $0.56 \mathrm{~nm}^{-2}$. However, in all cases, the magnitude of the electrostatic potential is overall lower for PMKE (and PSKE, albert not at $0.37 \mathrm{~nm}^{-2}$ ) than for the corresponding uncharged brush. It might be interesting to study if such a lowering of the potential could lessen the electrostatic interaction between the surface and charged residues in adsorbing proteins. 


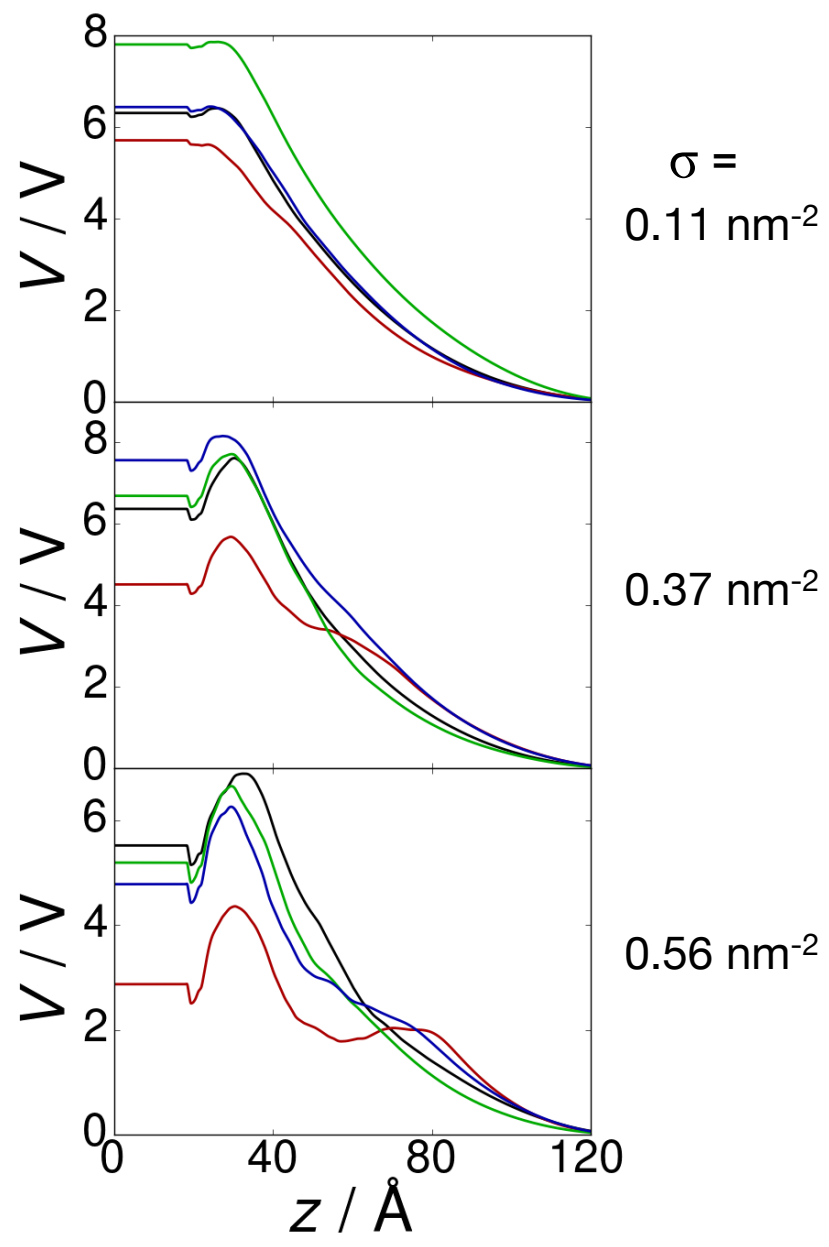

Figure 5 Electrostatic potential for (top to bottom) $\sigma=0.11 \mathrm{~nm}^{-2}, 0.37 \mathrm{~nm}^{-2}$, and $0.56 \mathrm{~nm}^{-2}$ brushes. Black, red, green, and blues lines denote PM, PMKE, PS, and PSKE brushes respectively.

We calculated the root-mean-squared fluctuations (RMSF) of each residue to characterize the mobility of the different peptoid species (Figure 6). For low to intermediate grafting densities (0.11 and $0.37 \mathrm{~nm}^{-2}$ ), the zwitterionic chains (PMKE and PSKE) have higher RMSF than the neutral chains (PM and PS). For all peptoids and chain densities, residues at the N-terminus (residue 0) are more mobile than those in the centre, likely due to the higher free volume 
available at the brush surface. Increasing the grafting density decreases the mobility of chains, due to the reduced free volume for the chains, and a difference between the charged and uncharged chains is no longer seen. Nonetheless the chains can all be considered fairly mobile even at the highest grafting density, with a RMSF greater than $2 \AA$ for all residues in all cases. A high mobility is considered to be necessary for brushes to act as an entropic barrier to the approach of proteins to the surface, thus preventing protein adsorption.

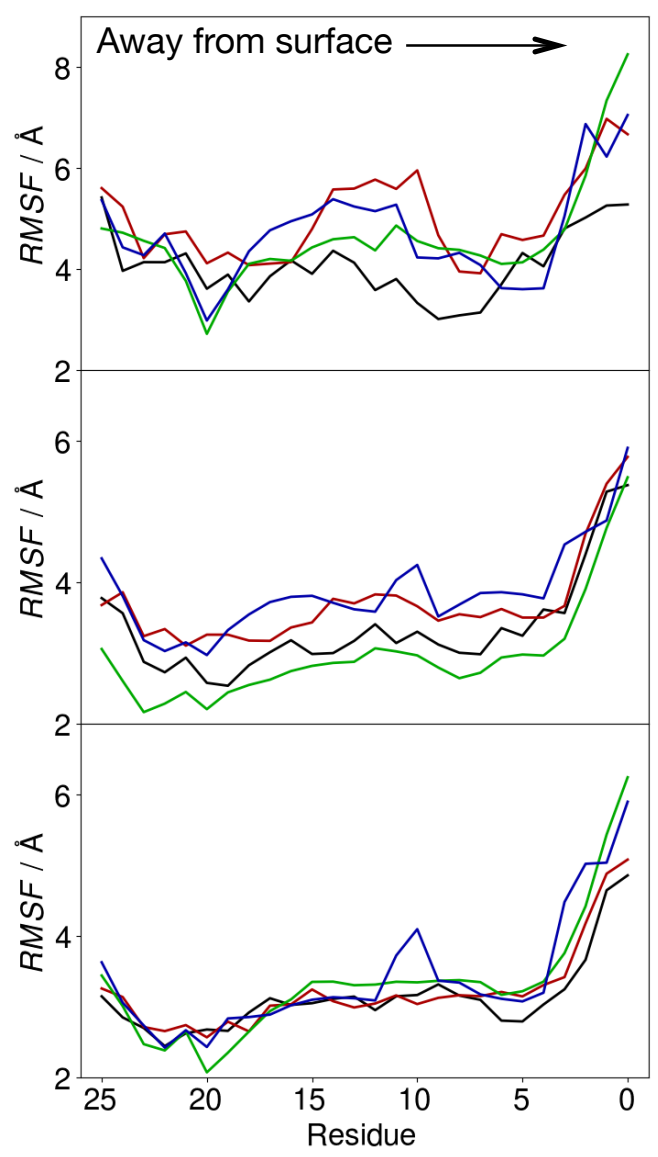

Figure 6 Average root mean-squared fluctuations (RMSF) per residue for (top to bottom) $\sigma=$ $0.11 \mathrm{~nm}^{-2}, 0.37 \mathrm{~nm}^{-2}$, and $0.56 \mathrm{~nm}^{-2}$ brushes. Black, red, green, and blues lines denote PM, PMKE, PS, and PSKE brushes respectively. Residues numbered from the N-terminal acetyl group (residue 0) to the C-terminal DOPA (residue 25) anchored on the surface. 


\subsection{Water structure and brush hydration}

The structure and dynamics of water near the surface plays a key role in determining the antifouling behaviour of a surface. Analysis of the number of hydrogen bonds that each residue forms with water molecules (Figure 7(a)) shows that every peptoid residues participate in hydrogen bonding, indicating a high degree of hydration of the brushes. Nme residues form slightly more hydrogen bonds than Sar residues, due to the oxygen atom in the methoxyethyl sidechain in addition to the $\mathrm{O}$ and $\mathrm{N}$ of each peptoid tertiary amide linkage. Typically each Nme residue forms slightly more than 2 hydrogen bonds with water molecules on average, consistent with previous simulation studies ${ }^{65}$ while each Sar forms slightly fewer $(\sim 1.5)$ H-bonds. The charged Nlys and Nglu residues for both PMKE and PSKE brushes form, respectively, 4 and 10 hydrogen bonds on average, significantly more than the uncharged residues (see also discussion of Figure 9 below).

The large number of $\mathrm{H}$-bonds with water for each residue suggests that the brush interior is quite hydrophilic. Water density profiles with height from surface, $\rho(\mathrm{z})$ (Figure 7(b)), for the different brushes show that there is a significant amount of water within the brushes. The water density within the PS/PSKE brushes is higher than for PM/PMKE brushes, consistent with the smaller volume of the Sar vs. Nme sidechains, but in all cases the brushes are significantly hydrated. This is consistent with a hydrophilic interior to the brushes, which has been shown to be advantageous for anti-fouling behavior. ${ }^{66}$ In all cases there are large peaks in the water density near the $\mathrm{TiO}_{2}$ surface, with the height of this peak decreasing as the chain density increases (see also ESI Figure S1). This is due to the ability of water to penetrate into the peptoid brush and the hydrophilic nature of the underlying $\mathrm{TiO}_{2}$ substrate. Similar peaks have been seen in previous simulations of water in contact with bare $\mathrm{TiO} 2$ surfaces. ${ }^{58,67}$ 


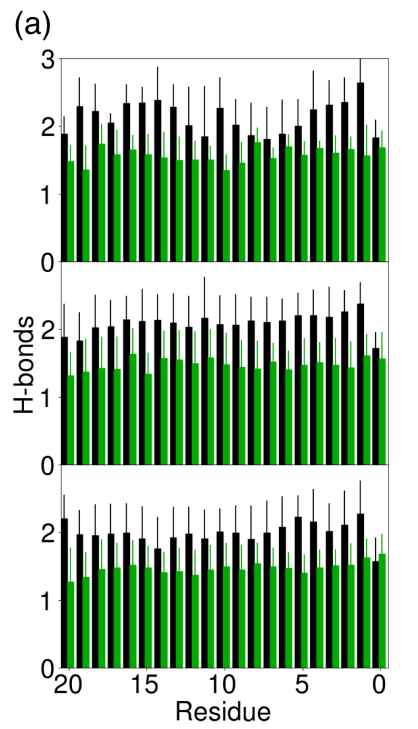

(b)

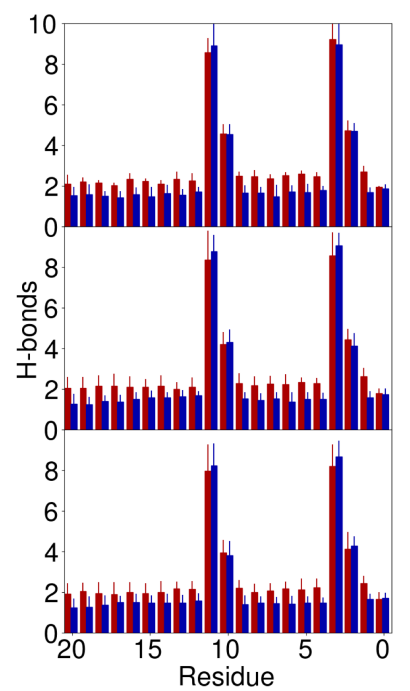

(c)
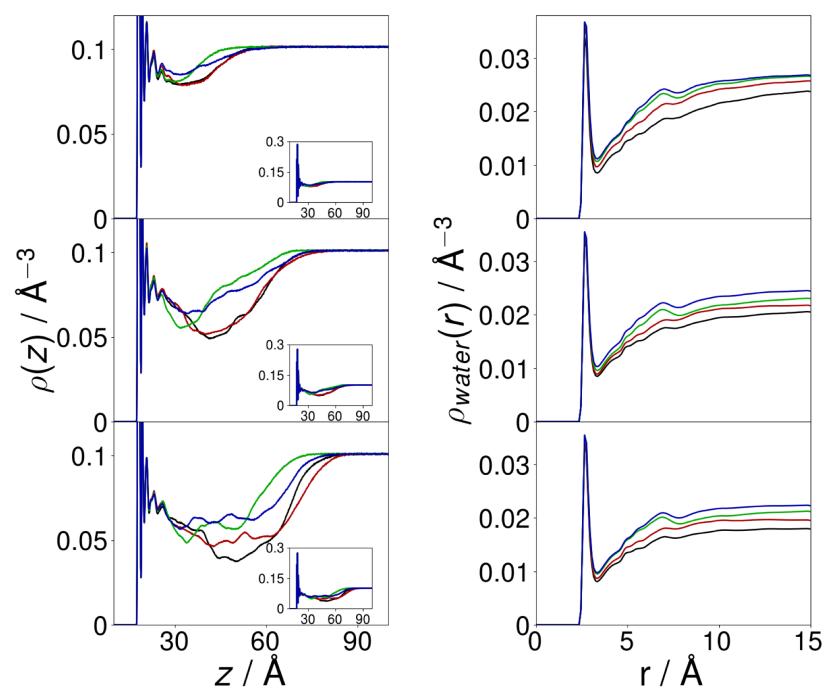

Figure 7 (a) Average number of hydrogen bonds for each residue in the peptoid segments for (top to bottom) $\sigma=0.11,0.37$, and $0.56 \mathrm{~nm}^{-2}$. Black, red, green, and blue denote PM, PMKE, PS, and PSKE brushes respectively. (b) Water density profiles for (top to bottom) $\sigma=0.11,0.37$, and $0.56 \mathrm{~nm}^{-2}$. See Figure S1 for full-scale graphs of the insets, which highlight the density of the adsorbed water on $\mathrm{TiO}_{2}$. Colours as in (a). (c) Radial water density around carbonyl oxygens for (top to bottom) $\sigma=0.11,0.37$, and $0.56 \mathrm{~nm}^{-2}$. Colours as in (a). 
The interaction of water molecules with the peptoids can be more closely examined through the water radial density profiles around the backbone carbonyl atoms (Figure 7(c)). The peaks at $\sim 3 \AA$ are essentially identical for the different brushes, indicating that water is strongly bound to the peptoid backbone, irrespective of the sidechains or surface density. Differences appear for the different brushes at larger separations. Consistent with Figure 7(b), the density of water about 10-15 $\AA$ away from the carbonyl O atoms for PM is the lowest among the brush species due to the larger sidechain of Nme. However, the water density is higher for the zwitterionic brushes than the uncharged counterparts, although this difference is greater for Nme based brushes.

The number of water molecules around each carbonyl oxygen can be quantified by integrating the radial density profiles

$$
N_{\text {water }}=4 \pi \int_{0}^{r_{c}} r^{2} \rho_{\text {water }}(r)
$$

where the integral is taken up to the second solvation shell $\left(r_{c}=7 \AA\right)$. This is higher for the zwitterionic peptoids than for their neutral equivalents (Table 2). The number of water molecules is also higher for the Sar based peptoids than the Nme peptoids, due to the lower side chain volumes. 
Table 2 Number of water molecules around carbonyl oxygen atoms for all residues $\left(N_{\text {water }}\right)$ and Nlys and $\mathrm{Nglu}\left(N_{\text {water }}\right.$ (Nlys) and $\left.N_{\text {water }}(\mathrm{Nglu})\right)$ residues or their equivalents in neutral peptoids.

\begin{tabular}{|l|l|l|l|l|}
\hline Peptoid & Density & $N_{\text {water }}$ & $N_{\text {water }}($ Nlys $)$ & $N_{\text {water }}(\mathrm{Nglu})$ \\
\hline \multirow{4}{*}{ PM } & $0.11 \mathrm{~nm}^{-2}$ & 21.32 & 21.41 & 20.92 \\
\cline { 2 - 5 } & $0.37 \mathrm{~nm}^{-2}$ & 20.50 & 20.17 & 19.94 \\
\cline { 2 - 5 } & $0.56 \mathrm{~nm}^{-2}$ & 19.48 & 18.27 & 17.22 \\
\hline PMKE & $0.11 \mathrm{~nm}^{-2}$ & 24.24 & 24.21 & 24.01 \\
\cline { 2 - 5 } & $0.37 \mathrm{~nm}^{-2}$ & 21.97 & 21.91 & 21.43 \\
\cline { 2 - 5 } & $0.56 \mathrm{~nm}^{-2}$ & 21.08 & 21.58 & 21.24 \\
\hline PS & $0.11 \mathrm{~nm}^{-2}$ & 26.59 & 26.09 & 26.24 \\
\cline { 2 - 5 } & $0.37 \mathrm{~nm}^{-2}$ & 23.92 & 24.68 & 24.17 \\
\cline { 2 - 5 } & $0.56 \mathrm{~nm}^{-2}$ & 23.49 & 24.68 & 23.63 \\
\hline PSKE & $0.11 \mathrm{~nm}^{-2}$ & 27.41 & 27.39 & 27.71 \\
\cline { 2 - 5 } & $0.37 \mathrm{~nm}^{-2}$ & 25.50 & 26.89 & 27.63 \\
\cline { 2 - 5 } & $0.56 \mathrm{~nm}^{-2}$ & 24.11 & 25.43 & 24.91 \\
\hline
\end{tabular}

The role of the charged Nglu and Nlys residues in binding water can be examined by comparing the structure of water around these residues with those at equivalent sequence positions in the neutral peptoids. While the radial density profiles (Figure 8) are relatively similar between the neutral and charged residues for the first peak (around $2 \AA$ ), there is more water beyond this around the charged residues than the equivalent neutral residues. The increased density of water may arise due to the strong attraction between the charged sidechains and water. At large distances the water density is higher for PMKE than PM, but similar for PS and PSKE. 
This is also reflected by the higher number of water molecules around the charged residues then in the neutral equivalents (Table 2).

The location of the charged residues on the outside of the zwitterionic brushes (Figure 3(a)) means that these may play a role in ordering water near the surface where proteins first interact with the brushes. Inspection of the RDFs between the charged atoms on the sidechains (N $\zeta$ on Nlys and Oع on Nglu) and water show tight ordering of water (Figure 9(a)). At low separation < $7 \AA$ there are a number peaks in the $\mathrm{N} \zeta$-water and Oع-water RDFs indicative of the hydrogen bonding between them and water molecules. The location and heights of the peaks for Nlys and Nglu are essentially the same for PMKE and PSKE, indicating that the local water structure around these residues is similar. The rest of the peptoid chains appear to have little effect on the water structure around the charged residues.

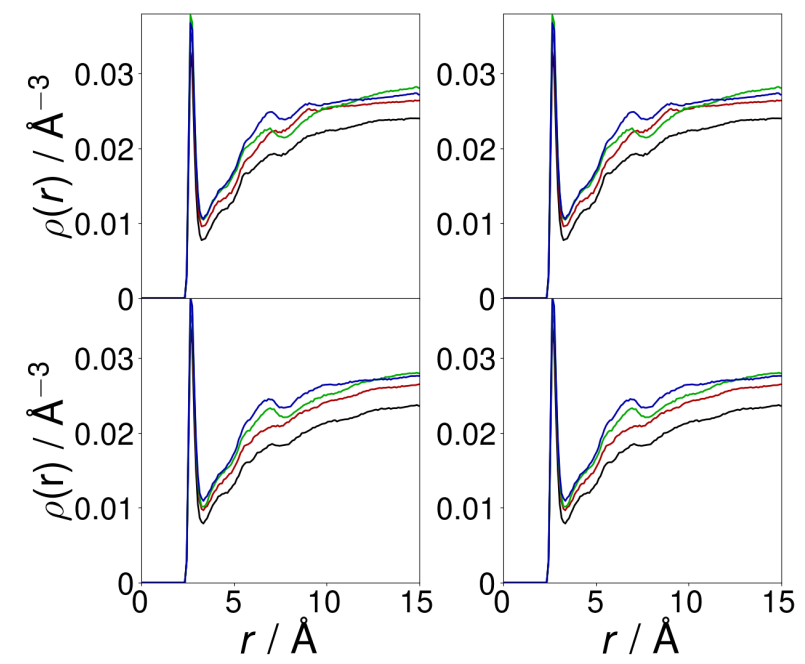

Figure 8 Comparison of water radial density profiles for charged and neutral peptoid residues. Top and bottom panels show $\sigma=0.11 \mathrm{~nm}^{-2}$ (top) and $\sigma=0.56 \mathrm{~nm}^{-2}$ (bottom). Left-hand panel shows Nlys and equivalent residues (residues 2 and 10) in neutral brushes, right-hand panel shows Nglu and equivalent residues (residues 3 and 11) in neutral brushes. Black, red, green, and blue denote PM, PMKE, PS, and PSKE respectively. 
(a)

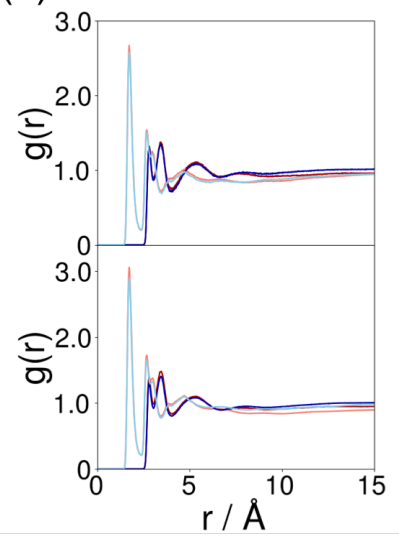

(b)

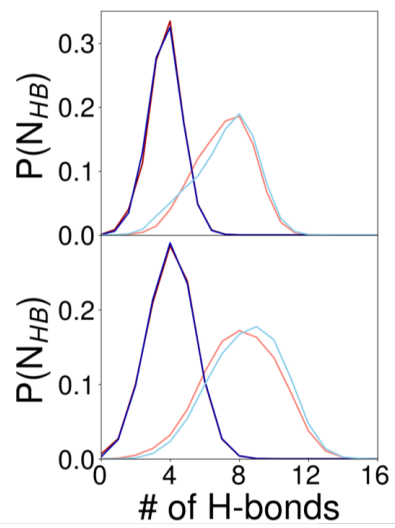

(c)

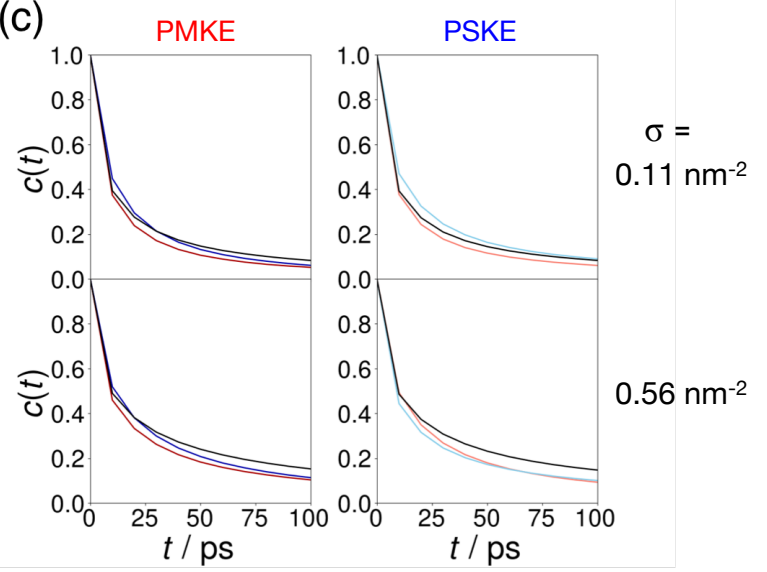

Figure 9 (a) RDFs between water molecules and charged residues for $\sigma=0.11$ (top) and $0.56 \mathrm{~nm}^{-}$

${ }^{2}$ (bottom). Red, pink, blue, and light blue denote Nlys (PMKE), Nglu (PMKE), Nlys (PSKE), and Nglu (PSKE). (b) Histograms of numbers of hydrogen bond for charged residues for $\sigma=$ 0.11 (top) and $0.56 \mathrm{~nm}^{-2}$ (bottom). Colours as in (a) (c) Hydrogen bond auto-correlation functions, $c(t)$, for PMKE $\sigma=0.11 \mathrm{~nm}^{-2}$ (top left), PMKE $\sigma=0.56 \mathrm{~nm}^{-2}$ (bottom left), PSKE $\sigma=$ $0.11 \mathrm{~nm}^{-2}$ (top right), PSKE $\sigma=0.56 \mathrm{~nm}^{-2}$ (bottom right). $c(t)$ is 1 if that pair of atoms forms a hydrogen bond and 0 otherwise. Blue, red, and black lines denote hydrogen bonds with Nlys residues, Nglu residues, and all residues respectively.

Furthermore, we have calculated the probability distribution of the numbers of hydrogen bonds $\left(P\left(\mathrm{~N}_{H \mathrm{~B}}\right)\right.$, Figure $\left.9(\mathrm{~b})\right)$ and the hydrogen bond autocorrelation function $(c(t)$, Figure $9(\mathrm{c}))$ for charged residues, to investigate the stability/dynamics of the water layer near the surface. Consistent with Figure 7(a), the probability distribution shows that Nglu residues form more hydrogen bonds with water than the Nlys residues regardless of whether these are on PMKE or PSKE chains. (There are two oxygens vs. one nitrogen as hydrogen bond acceptors in Nglu vs. Nlys sidechains). The characteristic relaxation time for H-bonding with the charged residues (the 
time over which $c(t)$ drops to $1 / \mathrm{e})$ is approximately $20 \mathrm{ps}$. This is similar to the previous simulation work of $\mathrm{Ham}^{65}$ but is longer than typical for hydrogen bonds between water molecules $\left(\sim 5.5 \mathrm{ps}^{65}\right)$, indicating that the hydrogen bonding of the brush with water is relatively persistent near the brush surface. When all residues throughout the brushes are considered, the autocorrelation functions assume slightly higher values, indicating longer decay times. This is due to the slower dynamics of water molecules trapped in the interior of the brush, which may further augment the hydration of the zwitterionic brushes.

\section{Discussion}

\subsection{Peptoid brush structure and chain flexibility}

The typical peptoid brush thickness is shown by MD to increase with grafting density (Figures 2 and 3), as also previously shown by molecular theory. ${ }^{49,}{ }^{50}$ Our MD results show that at intermediate to high densities $\left(0.37\right.$ and $\left.0.56 \mathrm{~nm}^{-2}\right)$, the zwitterionic peptoids form a well-defined layer with relatively uniform thickness and top surface. In particular, the charged residues enable the PSKE brush with the smaller Sar "background" residue to become more uniform compared with its uncharged PS counterpart.

All the brush species display a high degree of mobility, as measured through the root meansquared fluctuations of the peptoid residues (Figure 6). The mobilities of the zwitterionic brushes are even higher than the uncharged counterparts at 0.11 and $0.37 \mathrm{~nm}^{-2}$ chain densities, although this difference is suppressed at a higher chain density $\left(0.56 \mathrm{~nm}^{-2}\right)$. A highly flexible brush layer may be expected to promote resistance against protein adsorption.

Interestingly, the zwitterionic brushes (PMKE and PSKE) adopt configurations that preferentially present the charged peptoid residues on the exterior of the brush surface (Figures 2 
and 3), notwithstanding the fact that the poly( $N$-substituted glycine) peptoid backbone itself is also polar and highly hydrophilic. ${ }^{50}$ While the end-to-end chain lengths $\left(r_{\mathrm{ee}}\right)$ of PM and PMKE, which have similar molecular volumes, are also similar, PSKE with the lower molecular volume also adopt $r_{\mathrm{ee}}$ close to that of PMKE (Figure 3(b)). In contrast, the underlying uncharged PS design adopt significantly shorter $r_{\text {ee }}$. Thus the zwitterionic residues appear to dominate the chain behaviour when present and contribute to extended brush conformations. The presence of the charged groups of opposite sign on the surface acts to minimize the dipole of the surface, enhancing the surface hydration while possibly also suppressing electrostatic interactions with proteins (see also section 4.2), as seen in previous work on self-assembled monolayers. ${ }^{34,68}$ In these previous cases, however, the oppositely charged units are found to form into well-ordered arrays, unlike the fluctuating/disordered chain structures seen in our simulations (Figure 2).

At lower grafting densities, the uncharged PS chains with smaller molecular volume tend to leave voids in the brush structure (Figure 2), but the chains are also observed to be more able to adopt lateral extended conformations (Figure 3(c)) that (at least partially) span these voids, perhaps due to the larger free volume available in these layers. This could contribute to the similar critical brush densities for preventing protein adsorption observed experimentally for both PS and PM brushes. ${ }^{18,50}$

\subsection{Charge effects and water structure}

We have characterized the H-bonding associated with each residue along the peptoid sequences as well as with the charged sidechains (Figures 7 to 9). All the peptoid brushes are highly hydrated, containing a significant amount of water. We also observe significant charge complexation and neutralization of the oppositely charged groups on PMKE and PSKE, as characterized by the RDF involving oppositely charged atoms on the brush sidechains, especially 
at lower chain densities (Figure 4). Moreover, the calculated electric potential profiles for the zwitterionic brushes, which include also partial charges of all groups, are similar or lower than the corresponding uncharged brushes, notwithstanding slight protrusions of the profiles near the brush surface due to segregation of the charged residues (Figure 5). Interestingly, the PMKE profile compared to all other brushes is roughly reduced by half, and might correlate with lower electrostatic interactions with incoming proteins.

Furthermore, the radial density profile of water with backbone carbonyl oxygen atoms and hydrogen bonding groups in sidechains (Figures 8 and 9) show high amounts of water around these polar groups, which also show reduced dynamics. The structuring of water (kosmotropicity) has been identified in previous studies. ${ }^{69,70}$ The water radial density profile involving backbone carbonyl oxygens also show the highest amounts of water around PSKE chains (Figure 7), the zwitterionic peptoid with the lower molecular volume that can accommodate more water molecules within the brush. Overall long lived contacts between water molecules and the zwitterionic brushes, as measured by the autocorrelation decay time of hydrogen bonds (Figure 9), is significantly longer than between free water molecules.

Unlike surfaces such as self-assembled monolayers, the diffuse nature of the brush surface does not lead to significant ordering of water molecules, even though ordering is observed at the smaller length scale around individual charge/polar groups of the peptoid chains. This can be seen by the smoothness of the water density profiles near the surface. Examination of the number of peptoid-water hydrogen bond profiles (Figure 10) also shows this lack of ordering at the brush surface. For the uncharged PM and PS brushes the peptoid-water hydrogen bond profile essentially follows the peptoid brush density profile. For the PMKE and PSKE there is an increase in the number of hydrogen bonds per residue near the brush surface; this is due to the 
concentration of Nlys and Nglu residues, which have more hydrogen bonding groups than the neutral residues, partitioning towards the brush surface. In neither case are significant differences seen between the behaviour of water molecules at the brush surface and those within the brush.
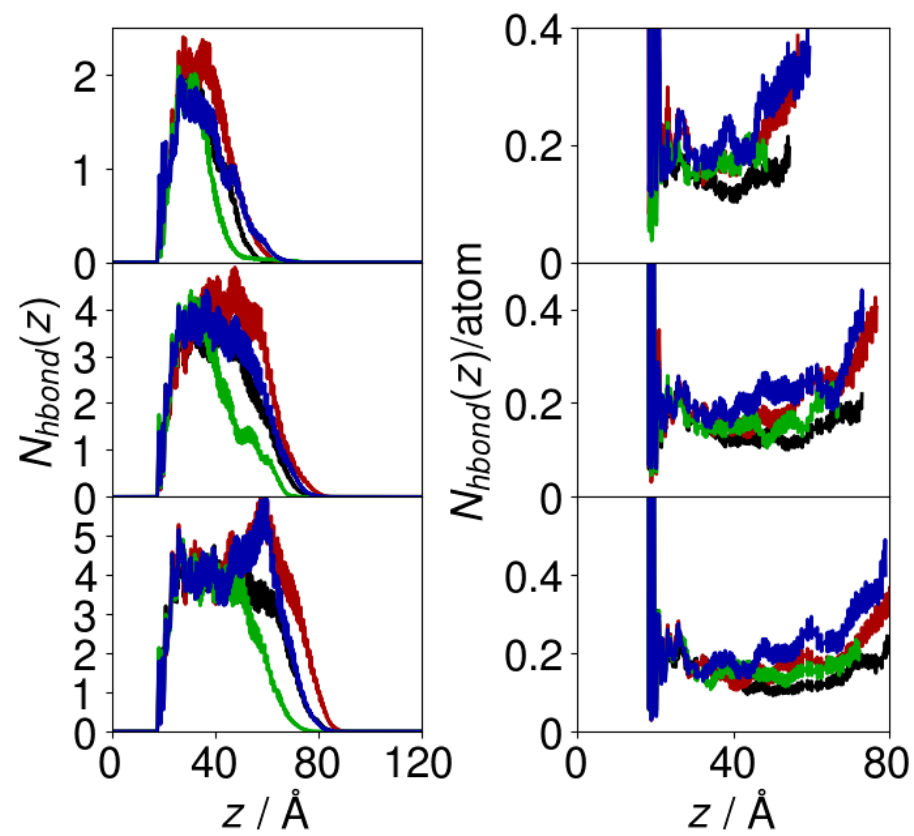

Figure 10 Number of peptoid-water hydrogen bonds (left) and number of peptoid-water hydrogen bonds normalized by number of peptoid atoms (right) as a function of z. PM, PMKE, PS, and PSKE denoted by black, red, green, and blue lines respectively. 


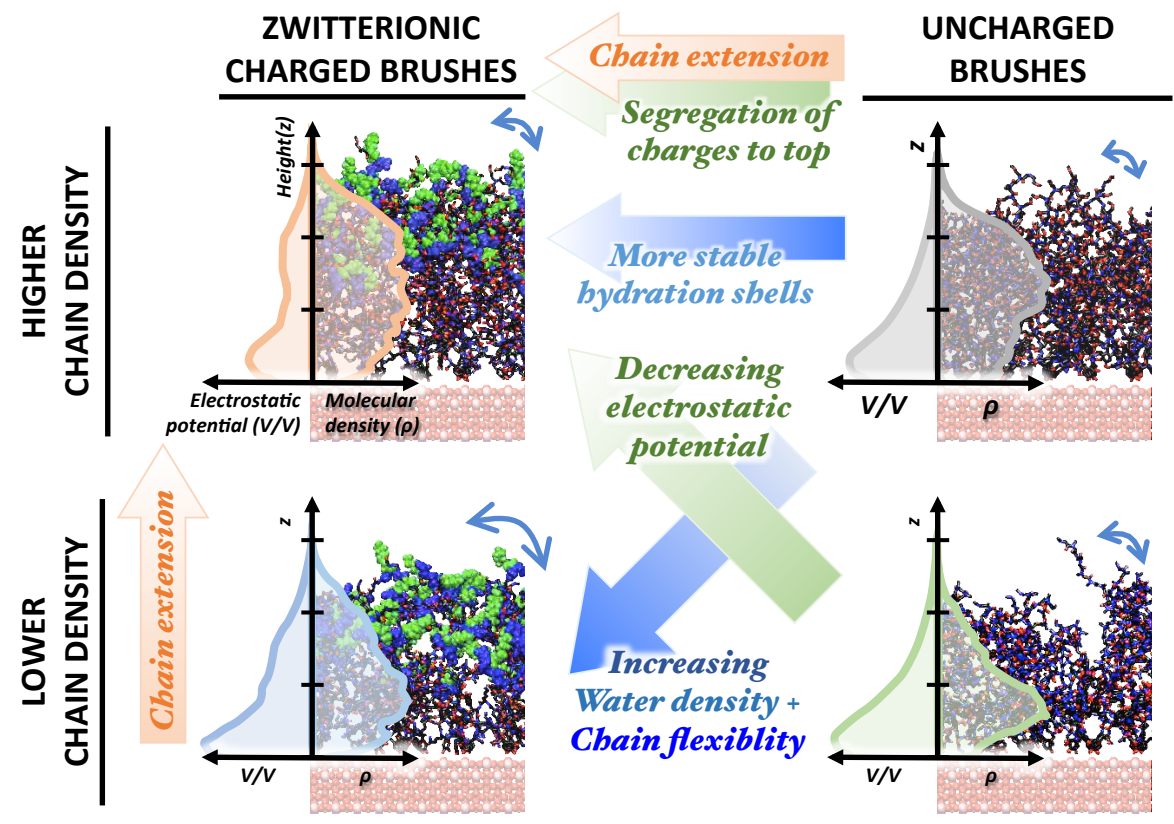

Figure 11. Schematic illustrating the balance between charge, hydration, chain density and molecular volume effects for the zwitterionic peptoid brush system studied.

\section{Conclusion}

The excellent antifouling properties of zwitterionic brushes have been well reported. While it is unsurprising that zwitterionic groups may confer greatly to hydration of the hydrophobic backbones of polyvinyl polymers grafted by surface initiated polymerization, this MD study shows that the contribution by pairs of oppositely charged residues to hydration around natively hydrophilic chains is also highly significant. Where present, the zwitterionic pairs play major roles in hydrogen bonding, structuring, and the stability of water around the peptoid brush chains studied. This MD study also investigated the balance between charge, hydration, and molecular volume/chain density effects. The principal effects are illustrated in Figure 11. The zwitterionic pairs significantly promote extended chain conformations further than by lateral chain compression as chain density increases and regardless of sidechain molecular volume. Charge 
complexation, or "self-association", ${ }^{71-74}$ of the charged sidechains is especially significant at "low" chain densities but still leads to lowered electrostatic potentials of the zwitterionic brushes compared to their uncharged (but polar) counterparts at all chain densities. Indeed, we emphasize in this study a highly dynamic nature of such transient charge complexation. Some properties promoted by the charged residues, such as chain flexibility and the water density around chains, actually increase more prominently at "low" and "intermediate" chain densities, and are suppressed by free volume effects as chain densities increase further.

Antifouling polymer brushes are considered to require high degrees of hydration, extended chain conformations, as well as high chain flexibility. ${ }^{32,65}$ Our computational study shows in detail how a zwitterionic charge design promotes these properties. Experimental data reported in the literature shows that the relatively short peptoids modelled require high grafting densities to enable good antifouling properties. ${ }^{18,49,50}$ However, the "low" density $\left(0.11 \mathrm{~nm}^{-2}\right)$ condition in our current simulations may nonetheless be relevant for antifouling brushes in general since it could correspond more closely to higher molecular weight polydisperse brushes grafted by surface-initiated polymerization. In fact, at higher chain densities, we show that some of the effects conferred by the charged residues are suppressed by the molecular volume taken up by peptoid chains (of uniform chain lengths) at high grafting density, as well as by larger sidechains if present. Although higher chain densities generally promote antifouling properties, at low, intermediate or polydisperse polymer densities, or when high grafting densities are not available, it is conceivable that zwitterionic designs could be (especially) advantageous. Future protein adsorption studies, by computation or experiments, that directly investigate the interplay between chain density, hydration, and zwitterionic charge effects could provide a fruitful avenue of research to improve resistance against biofouling. 
Supporting Information. Simulation input files (initial conformations in gromacs gro format, gromacs topology files, force field parameter files) and video representations of the peptoid brush simulations are available free of charge at http://pubs.acs.org.

\section{AUTHOR INFORMATION}

Corresponding Authors

*Email: david.cheung@nuigalway.ie

*Email: aaron.lau@strath.ac.uk

ORCID

D. L. Cheung: 0000-0002-3994-2295

K. H. A. Lau: 0000-0003-3676-9228

Notes

The authors declare no competing financial interest.

Author Contributions

The manuscript was written through contributions of all authors. All authors have given approval to the final version of the manuscript.

\section{ACKNOWLEDGMENT}


We wish to acknowledge the SFI/HEA Irish Centre for High-End Computing (ICHEC) for the provision of computational facilities and support. KHAL also acknowledges support by EPSRC UK (grant EP/P026265/1). Coordinates for the rutile slab were provided by Dr. Natalia Martsinovich (University of Sheffield). The research data associated with this paper will become available at the following link: http://dx.doi.org/10.15129/f77ddee17-9832-4263-bc52400d0a41f72f.

\section{REFERENCES}

(1) Blaszykowski, C.; Sheikh, S.; Thompson, M., Surface chemistry to minimize fouling from blood-based fluids. Chemical Society Reviews 2012, 41, 5599-5612.

(2) Ci, J. L.; Kang, H. L.; Liu, C. G.; He, A. H.; Liu, R. G., Protein Resistance Adsorption Mechanism and Applications of Zwitterionic Polymers. Prog. Chem. 2015, 27, 1198-1212.

(3) Ayres, N., Polymer brushes: Applications in biomaterials and nanotechnology. Polym. Chem. 2010, 1, 769-777.

(4) Halperin, A., Polymer Brushes that Resist Adsorption of Model Proteins:\&nbsp; Design Parameters. Langmuir 1999, 15, 2525-2533.

(5) McPherson, T.; Kidane, A.; Szleifer, I.; Park, K., Prevention of Protein Adsorption by Tethered Poly(ethylene oxide) Layers: Experiments and Single-Chain Mean-Field Analysis. Langmuir 1998, 14, 176-186.

(6) Satulovsky, J.; Carignano, M. A.; Szleifer, I., Kinetic and thermodynamic control of protein adsorption. Proceedings of the National Academy of Sciences 2000, 97, 9037-9041. 
(7) Ishihara, K.; Nomura, H.; Mihara, T.; Kurita, K.; Iwasaki, Y.; Nakabayashi, N., Why do phospholipid polymers reduce protein adsorption? Journal of Biomedical Materials Research 1998, 39, 323-330.

(8) Ostuni, E.; Chapman, R. G.; Holmlin, R. E.; Takayama, S.; Whitesides, G. M., A Survey of Structure-Property Relationships of Surfaces that Resist the Adsorption of Protein. Langmuir 2001, 17, 5605-5620.

(9) Ostuni, E.; Chapman, R. G.; Liang, M. N.; Meluleni, G.; Pier, G.; Ingber, D. E.; Whitesides, G. M., Self-Assembled Monolayers That Resist the Adsorption of Proteins and the Adhesion of Bacterial and Mammalian Cells. Langmuir 2001, 17, 6336-6343.

(10) Halperin, A.; Krol’’’ger, M., Ternary Protein Adsorption onto Brushes: Strong versus Weak. Langmuir 2009, 25, 11621-11634.

(11) Huang, K. T.; Fang, Y. L.; Hsieh, P. S.; Li, C. C.; Dai, N. T.; Huang, C. J., Zwitterionic nanocomposite hydrogels as effective wound dressings. J. Mat. Chem. B 2016, 4, 4206-4215.

(12) Huang, C. J.; Mi, L.; Jiang, S. Y., Interactions of alginate-producing and -deficient Pseudomonas aeruginosa with zwitterionic polymers. Biomaterials 2012, 33, 3626-3631.

(13) Jiang, S. Y.; Cao, Z. Q., Ultralow-Fouling, Functionalizable, and Hydrolyzable Zwitterionic Materials and Their Derivatives for Biological Applications. Adv. Mater. 2010, 22, 920-932.

(14) Yoshimoto, K.; Hirase, T.; Madsen, J.; Armes, S. P.; Nagasaki, Y., Non-Fouling Character of Poly[2-(methacryloyloxy)ethyl Phosphorylcholine]-Modified Gold Surfaces 
Fabricated by the 'Grafting to' Method: Comparison of its Protein Resistance with Poly(ethylene glycol)-Modified Gold Surfaces. Macromolecular Rapid Communications 2009, 30, 2136-2140.

(15) Chen, S. F.; Jiang, S. Y., A new avenue to nonfouling materials. Adv. Mater. 2008, 20, $335-+$.

(16) Tada, S.; Inaba, C.; Mizukami, K.; Fujishita, S.; Gemmei-Ide, M.; Kitano, H.; Mochizuki, A.; Tanaka, M.; Matsunaga, T., Anti-Biofouling Properties of Polymers with a Carboxybetaine Moiety. Macromol Biosci 2009, 9, 63-70.

(17) Ishihara, K.; Oshida, H.; Endo, Y.; Ueda, T.; Watanabe, A.; Nakabayashi, N., Hemocompatibility of Human Whole-Blood on Polymers with a Phospholipid Polar Group and Its Mechanism. Journal of Biomedical Materials Research 1992, 26, 1543-1552.

(18) Lau, K. H. A.; Sileika, T. S.; Park, S. H.; Sousa, A. M. L.; Burch, P.; Szleifer, I.; Messersmith, P. B., Molecular Design of Antifouling Polymer Brushes Using Sequence-Specific Peptoids. Adv. Mater. Interf. 2015, 2, 1400225.

(19) Bernards, M. T.; Cheng, G.; Zhang, Z.; Chen, S. F.; Jiang, S. Y., Nonfouling polymer brushes via surface-initiated, two-component atom transfer radical polymerization. Macromolecules 2008, 41, 4216-4219.

(20) Nowinski, A. K.; Sun, F.; White, A. D.; Keefe, A. J.; Jiang, S. Y., Sequence, Structure, and Function of Peptide Self-Assembled Monolayers. Journal of the American Chemical Society 2012, 134, 6000-6005. 
(21) Zhang, Z.; Chen, S.; Jiang, S., Dual-Functional Biomimetic Materials:\&nbsp; Nonfouling Poly(carboxybetaine) with Active Functional Groups for Protein Immobilization. Biomacromolecules 2006, 7, 3311-3315.

(22) Mi, L.; Bernards, M. T.; Cheng, G.; Yu, Q.; Jiang, S., pH responsive properties of nonfouling mixed-charge polymer brushes based on quaternary amine and carboxylic acid monomers. Biomaterials 2010, 31, 2919-2925.

(23) Brault, N. D.; Sundaram, H. S.; Li, Y. T.; Huang, C. J.; Yu, Q. M.; Jiang, S. Y., Dry Film Refractive Index as an Important Parameter for Ultra-Low Fouling Surface Coatings. Biomacromolecules 2012, 13, 589-593.

(24) Yu, K.; Andruschak, P.; Yeh, H. H.; Grecov, D.; Kizhakkedathu, J. N., Influence of dynamic flow conditions on adsorbed plasma protein corona and surface-induced thrombus generation on antifouling brushes. Biomaterials 2018, 166, 79-95.

(25) Xie, Y.; Chen, L. T.; Zhang, X.; Chen, S. Q.; Zhang, M.; Zhao, W. F.; Sun, S. D.; Zhao, C. S., Integrating zwitterionic polymer and Ag nanoparticles on polymeric membrane surface to prepare antifouling and bactericidal surface via Schiff-based layer-by-layer assembly. J. Colloid Interface Sci. 2018, 510, 308-317.

(26) van Andel, E.; de Bus, I.; Tijhaar, E. J.; Smulders, M. M. J.; Savelkoul, H. F. J.; Zuilhof, H., Highly Specific Binding on Antifouling Zwitterionic Polymer-Coated Microbeads as Measured by Flow Cytometry. ACS Appl. Mater. Interfaces 2017, 9, 38211-38221. 
(27) Zhao, X. Z.; He, C. J., Efficient Preparation of Super Antifouling PVDF Ultrafiltration Membrane with One Step Fabricated Zwitterionic Surface. ACS Appl. Mater. Interfaces 2015, 7 , 17947-17953.

(28) Higaki, Y.; Nishida, J.; Takenaka, A.; Yoshimatsu, R.; Kobayashi, M.; Takahara, A., Versatile inhibition of marine organism settlement by zwitterionic polymer brushes. Polym. $J$. 2015, $47,811-818$.

(29) Davenport, D. M.; Lee, J.; Elimelech, M., Efficacy of antifouling modification of ultrafiltration membranes by grafting zwitterionic polymer brushes. Sep. Purif. Technol. 2017, 189, 389-398.

(30) Du, Y.; Gao, J. Y.; Chen, T. T.; Zhang, C.; Ji, J.; Xu, Z. K., Understanding the Oxidative Stability of Antifouling Polymer Brushes. Langmuir 2017, 33, 7298-7304.

(31) Feng, W.; Zhu, S. P.; Ishihara, K.; Brash, J. L., Protein resistant surfaces: Comparison of acrylate graft polymers bearing oligo-ethylene oxide and phosphorylcholine side chains. Biointerphases 2006, 1, 50-60.

(32) Chen, S. F.; Li, L. Y.; Zhao, C.; Zheng, J., Surface hydration: Principles and applications toward low-fouling/nonfouling biomaterials. Polymer 2010, 51, 5283-5293.

(33) Zhao, C.; Zhao, J.; Li, X. S.; Wu, J.; Chen, S. F.; Chen, Q.; Wang, Q. M.; Gong, X.; Li, L. Y.; Zheng, J., Probing structure-antifouling activity relationships of polyacrylamides and polyacrylates. Biomaterials 2013, 34, 4714-4724. 
(34) Chen, S. F.; Zheng, J.; Li, L. Y.; Jiang, S. Y., Strong resistance of phosphorylcholine self-assembled monolayers to protein adsorption: Insights into nonfouling properties of zwitterionic materials. Journal of the American Chemical Society 2005, 127, 14473-14478.

(35) Morisaku, T.; Watanabe, J.; Konno, T.; Takai, M.; Ishihara, K., Hydration of phosphorylcholine groups attached to highly swollen polymer hydrogels studied by thermal analysis. Polymer 2008, 49, 4652-4657.

(36) Xiao, S. W.; Zhang, Y. X.; Shen, M. X.; Chen, F.; Fan, P.; Zhong, M. Q.; Ren, B. P.; Yang, J. T.; Zheng, J., Structural Dependence of Salt-Responsive Polyzwitterionic Brushes with an Anti-Polyelectrolyte Effect. Langmuir 2018, 34, 97-105.

(37) Du, H. B.; Qian, X. H., The Hydration Properties of Carboxybetaine Zwitterion Brushes. J. Comput. Chem. 2016, 37, 877-885.

(38) Weers, J. G.; Rathman, J. F.; Axe, F. U.; Crichlow, C. A.; Foland, L. D.; Scheuing, D. R.; Wiersema, R. J.; Zielske, A. G., Effect of the Intramolecular Charge Separation Distance on the Solution Properties of Betaines and Sulfobetaines. Langmuir 1991, 7, 854-867.

(39) Knight, A. S.; Zhou, E. Y.; Francis, M. B.; Zuckermann, R. N., Sequence Programmable Peptoid Polymers for Diverse Materials Applications. Adv. Mater. 2015, 27, 5665-5691.

(40) Lau, K. H. A., Peptoids for biomaterials science. Biomaterials Science 2014, 2, 627-633.

(41) El Yaagoubi, M.; Tewari, K. M.; Lau, K. H. A., Peptoid self-assembly and opportunities for creating protein-mimetic biomaterials and biointerfaces. In Self-assembling Biomaterials, Azevedo, H. S.; Silva, R. M. P. d., Eds. Elsevier-Woodhead Publishing: 2018; pp 95-112. 
(42) Murnen, H. K.; Rosales, A. M.; Dobrynin, A. V.; Zuckermann, R. N.; Segalman, R. A., Persistence length of polyelectrolytes with precisely located charges. Soft Matter 2013, 9, 90-98.

(43) Zuckermann, R. N.; Kerr, J. M.; Kent, S. B. H.; Moos, W. H., Efficient method for the preparation of peptoids [oligo(N-substituted glycines)] by submonomer solid-phase synthesis. Journal of the American Chemical Society 1992, 114, 10646-10647.

(44) Rosales, A. M.; Segalman, R. A.; Zuckermann, R. N., Polypeptoids: a model system to study the effect of monomer sequence on polymer properties and self-assembly. Soft Matter 2013, $9,8400-8414$.

(45) Sternhagen, G. L.; Gupta, S.; Zhang, Y. H.; John, V.; Schneider, G. J.; Zhang, D. H., Solution Self-Assemblies of Sequence-Defined Ionic Peptoid Block Copolymers. Journal of the American Chemical Society 2018, 140, 4100-4109.

(46) Park, S. H.; Szleifer, I., Structural and Dynamical Characteristics of Peptoid Oligomers with Achiral Aliphatic Side Chains Studied by Molecular Dynamics Simulation. The Journal of Physical Chemistry B 2011, 115, 10967-10975.

(47) Mirijanian, D. T.; Mannige, R. V.; Zuckermann, R. N.; Whitelam, S., Development and Use of an Atomistic CHARMM-Based Forcefield for Peptoid Simulation. J. Comput. Chem. 2014, 35, 360-370.

(48) Statz, A. R.; Meagher, R. J.; Barron, A. E.; Messersmith, P. B., New Peptidomimetic Polymers for Antifouling Surfaces. Journal of the American Chemical Society 2005, 127, 79727973. 
(49) Lau, K. H. A.; Ren, C.; Park, S. H.; Szleifer, I.; Messersmith, P. B., An ExperimentalTheoretical Analysis of Protein Adsorption on Peptidomimetic Polymer Brushes. Langmuir 2012, 28, 2288-2298.

(50) Lau, K. H. A.; Ren, C.; Sileika, T. S.; Park, S. H.; Szleifer, I.; Messersmith, P. B., Surface-Grafted Polysarcosine as a Peptoid Antifouling Polymer Brush. Langmuir 2012, 28, 16099-16107.

(51) Leng, C.; Buss, H. G.; Segalman, R. A.; Chen, Z., Surface Structure and Hydration of Sequence-Specific Amphiphilic Polypeptoids for Antifouling/Fouling Release Applications. Langmuir 2015, 31, 9306-9311.

(52) Schneider, M.; Tang, Z.; Richter, M.; Marschelke, C.; Forster, P.; Wegener, E.; Amin, I.; Zimmermann, H.; Scharnweber, D.; Braun, H. G.; Luxenhofer, R.; Jordan, R., Patterned Polypeptoid Brushes. Macromol Biosci 2016, 16, 75-81.

(53) Ryu, J. Y.; Song, I. T.; Lau, K. H. A.; Messersmith, P. B.; Yoon, T.-Y.; Lee, H., New Antifouling Platform Characterized by Single-Molecule Imaging. ACS Appl. Mater. Interfaces 2014, 6, 3553-3558.

(54) Soshee, A.; Zürcher, S.; Spencer, N. D.; Halperin, A.; Nizak, C., General In Vitro Method to Analyze the Interactions of Synthetic Polymers with Human Antibody Repertoires. Biomacromolecules 2014, 15, 113-121.

(55) MacKerell, A. D.; Bashford, D.; Dunbrack, R. L.; Evanseck, J. D.; Field, M. J.; Fischer, S.; Gao, J.; Guo, H.; Ha, S.; Joseph-McCarthy, D.; Kuchnir, L.; Kuczera, K.; Lau, F. T. K.; Mattos, C.; Michnick, S.; Ngo, T.; Nguyen, D. T.; Prodhom, B.; Reiher, W. E.; Roux, B.; 
Schlenkrich, M.; Smith, J. C.; Stote, R.; Straub, J.; Watanabe, M.; Wiórkiewicz-Kuczera, J.; Yin, D.; Karplus, M.; Dunbrack, R. L.; Evanseck, J. D.; Field, M. J.; Fischer, S.; Gao, J.; Guo, H.; Ha, S.; Joseph-McCarthy, D.; Kuchnir, L.; Kuczera, K.; Lau, F. T. K.; Mattos, C.; Michnick, S.; Ngo, T.; Nguyen, D. T.; Prodhom, B.; Reiher, W. E.; Roux, B.; Schlenkrich, M.; Smith, J. C.; Stote, R.; Straub, J.; Watanabe, M.; Wiórkiewicz-Kuczera, J.; Yin, D.; Karplus, M., All-Atom Empirical Potential for Molecular Modeling and Dynamics Studies of Proteins $\uparrow$. The Journal of Physical Chemistry B 1998, 102, 3586-3616.

(56) Gfeller, D.; Michielin, O.; Zoete, V., SwissSidechain: A molecular and structural database of non-natural sidechains. Nucleic Acids Research 2013, 41, 327-332.

(57) Mark, P.; Nilsson, L., Structure and dynamics of the TIP3P, SPC, and SPC/E water models at 298 K. Journal of Physical Chemistry A 2001, 105, 9954-9960.

(58) Predota, M.; Bandura, a. V.; Cummings, P. T.; Kubicki, J. D.; Wesolowski, D. J.; Chialvo, a. a.; Machesky, M. L.; Pr, M., Electric Double Layer at the Rutile ( 110 ) Surface . 1. Structure of Surfaces and Interfacial Water from Molecular Dynamics by Use of ab Initio Potentials Electric Double Layer at the Rutile ( 110 ) Surface . 1. Structure of Surfaces and Interfacial Wa. J. Phys. Chem. B 2004, 108, 12049-12060.

(59) Essmann, U.; Perera, L.; Berkowitz, M. L.; Darden, T.; Lee, H.; Pedersen, L. G., A smooth particle mesh Ewald method. J. Chem. Phys. 1995, 103, 8577.

(60) Van Der Spoel, D.; Lindahl, E.; Hess, B.; Groenhof, G.; Mark, A. E.; Berendsen, H. J. C., GROMACS: Fast, flexible, and free. J. Comput. Chem. 2005, 26, 1701-1718. 
(61) Hess, B.; Kutzner, C.; van der Spoel, D.; Lindahl, E., GROMACS 4: Algorithms for Highly Efficient, Load-Balanced, and Scalable Molecular Simulation. J. Chem. Theory Comput. 2008, 4, 435-447.

(62) Bussi, G.; Donadio, D.; Parrinello, M., Canonical sampling through velocity rescaling. $J$. Chem. Phys. 2007, 126, 014101/1-7.

(63) Michaud-Agrawal, N.; Denning, E. J.; Woolf, T. B.; Beckstein, O., MDAnalysis: A Toolkit for the Analysis of MolecularDynamics Simulations. J. Comput. Chem. 2011, 32, 23192327.

(64) Humphrey, W.; Dalke, A.; Schulten, K., VMD: Visual molecular dynamics. Journal of Molecular Graphics 1996, 14, 33-38.

(65) Ham, H. O.; Park, S. H.; Kurutz, J. W.; Szleifer, I. G.; Messersmith, P. B., Antifouling Glycocalyx-Mimetic Peptoids. Journal of the American Chemical Society 2013, 135, 1301513022.

(66) Herrwerth, S.; Eck, W.; Reinhardt, S.; Grunze, M., Factors that determine the protein resistance of oligoether self-assembled monolayers - Internal hydrophilicity, terminal hydrophilicity, and lateral packing density. Journal of the American Chemical Society 2003, 125, 9359-9366.

(67) Skelton, A. A.; Walsh, T. R., Interaction of liquid water with the rutile TiO2 (110) surface. Molecular Simulation 2007, 33, 379-389. 
(68) Holmlin, R. E.; Chen, X. X.; Chapman, R. G.; Takayama, S.; Whitesides, G. M., Zwitterionic SAMs that resist nonspecific adsorption of protein from aqueous buffer. Langmuir 2001, 17, 2841-2850.

(69) Zheng, J.; Li, L.; Chen, S.; Jiang, S., Molecular simulation study of water interactions with oligo (ethylene glycol)-terminated alkanethiol self-assembled monolayers. Langmuir 2004, 20, 8931-8938.

(70) Sheikh, S.; Blaszykowski, C.; Nolan, R.; Thompson, D.; Thompson, M., On the hydration of subnanometric antifouling organosilane adlayers: A molecular dynamics simulation. J. Colloid Interface Sci. 2015, 437, 197-204.

(71) Azzaroni, O.; Brown, A. A.; Huck, W. T. S., UCST Wetting Transitions of Polyzwitterionic Brushes Driven by Self-Association. Angewandte Chemie International Edition 2006, 45, 1770-1774.

(72) Chevalier, Y.; Kamenka, N.; Chorro, M.; Zana, R., Aqueous Solutions of Zwitterionic Surfactants with Varying Carbon Number of the Intercharge Group. 3. Intermicellar Interactions. Langmuir 1996, 12, 3225-3232.

(73) Cheng, G.; Liao, M.; Zhao, D.; Zhou, J., Molecular Understanding on the Underwater Oleophobicity of Self-Assembled Monolayers: Zwitterionic versus Nonionic. Langmuir 2017, $33,1732-1741$.

(74) Liao, M.; Cheng, G.; Zhou, J., Underwater Superoleophobicity of Pseudozwitterionic SAMs: Effects of Chain Length and Ionic Strength. The Journal of Physical Chemistry C 2017, $121,17390-17401$. 